\title{
Investigation of large artery calcification in non-contrast computed tomography with small vessel disease, large artery atherosclerosis and stroke subtype in ischaemic stroke patients
}

\author{
Authors: Armaan Iqbal, ${ }^{A}$ Kirti Kain ${ }^{B}$ and Ahamad Hassan ${ }^{C}$
}

\section{Introduction}

Strokes are the second leading cause of death worldwide. Arterial calcification has been associated with an increased frequency of stroke. Arterial calcification increases vessel stiffness, which has been indicated to be linked with cerebral small vessel disease.,2 However the relation of large artery calcification to stroke subtype and progression is uncertain, despite its ability to be identified simply using a non-contrast computed tomography (CT). ${ }^{3}$ This study aims to investigate the association between large artery calcification with both small vessel disease and large artery atherosclerosis in ischaemic stroke patients. Also to further investigate the association between large artery calcification and stroke subtype, with a focus on lacunar strokes.

\section{Methods}

The setting for this study took place at the Leeds General Infirmary. Ischaemic stroke patients were recruited from the stroke wards and stroke outpatient departments, as well as wards containing outlier stroke patients, between October 2018 and February 2019. A total of 70 patients were recruited within this period. Patients were assessed to have capacity to consent to take part in the study by either a doctor or stroke nurse specialist. A visual assessment of patient non-contrast CT head by a consultant neurologist was used to check for the presence of cerebral large vessel calcification. Magnetic resonance imaging (MRI) or CT were used for small vessel disease scores, and ultrasound or angiography was used to determine large artery atherosclerosis scores, both of which were based on simple visual rating scales. Strokes were subtyped based on the modified Trial of Org 10172 in Acute Stroke Treatment (TOAST) criteria.

\section{Results and discussion}

The mean age in the study population was 67 years for males and 69 years for females. There were no significant difference found

Authors: ${ }^{A}$ University of Leeds; ${ }^{B}$ Leeds Institute of Cardiovascular and Metabolic Medicine; ' Leeds General Infirmary, UK between males and females in risk factors including obesity, blood pressure and diabetes. The prevalence of large artery calcification was found to be $50 \%$ in the study population. Univariate analysis found no significant association between large artery calcification with leukoaraiosis ( $p$-value 0.087 ), or with degree of large artery atherosclerosis as measured by ultrasound scan or angiography ( $p$-value 0.731). There was also a lack of association found between large artery calcification and stroke subtype, when analysing lacunar strokes in comparison with other ischaemic stroke subtypes ( $p$-value 0.718 ). However large artery calcification was found to be associated with hypertension ( $p$-value 0.038 ). The findings from the study indicate that large artery calcification is not related to a particular subtype of stroke, but may indicate a marker of generalised atherosclerotic disease. This is supported by the association of large artery calcification with hypertension, hypertension being a risk factor for various strokes, not just a particular subtype, similarly large artery calcification may reflect hypertension in this way.

\section{Conclusion}

In conclusion the study findings support the idea that large artery calcification reflects advanced vascular disease rather than a specific ischaemic stroke subtype or mechanism.

\section{Conflict of interest statement}

None declared.

\section{References}

1 Chen Y, Shen F, Liu J, Yang GY. Arterial stiffness and stroke: de-stiffening strategy, a therapeutic target for stroke. Stroke Vasc Neurol 2017;2:65-72.

2 Stroke Association. State of the nation stroke statistics. Stroke Association, 2018. www.stroke.org.uk/system/files/sotn_2018.pdf [Accessed 21 March 2019].

3 Wu XH, Chen XY, Wang LJ, Wong KS. Intracranial artery calcification and its clinical significance. J Clin Neurol 2016;12:253-61. 